\title{
The hidden societal cost of antibiotic resistance per antibiotic prescribed in the United States: an exploratory analysis
}

Constantinos I. Michaelidis ${ }^{1,5^{*}}$, Michael J. Fine ${ }^{2,3}$, Chyongchiou Jeng Lin ${ }^{4}$, Jeffrey A. Linder ${ }^{1}$, Mary Patricia Nowalk ${ }^{4}$, Ryan K. Shields ${ }^{2}$, Richard K. Zimmerman ${ }^{4}$ and Kenneth J. Smith ${ }^{2}$

\begin{abstract}
Background: Ambulatory antibiotic prescribing contributes to the development of antibiotic resistance and increases societal costs. Here, we estimate the hidden societal cost of antibiotic resistance per antibiotic prescribed in the United States.

Methods: In an exploratory analysis, we used published data to develop point and range estimates for the hidden societal cost of antibiotic resistance (SCAR) attributable to each ambulatory antibiotic prescription in the United States. We developed four estimation methods that focused on the antibiotic-resistance attributable costs of hospitalization, second-line inpatient antibiotic use, second-line outpatient antibiotic use, and antibiotic stewardship, then summed the estimates across all methods.

Results: The total SCAR attributable to each ambulatory antibiotic prescription was estimated to be $\$ 13$ (range: $\$ 3-\$ 95)$. The greatest contributor to the total SCAR was the cost of hospitalization ( $\$ 9 ; 69 \%$ of the total SCAR). The costs of second-line inpatient antibiotic use ( $\$ 1 ; 8 \%$ of the total SCAR), second-line outpatient antibiotic use ( $\$ 2 ; 15 \%$ of the total SCAR) and antibiotic stewardship ( $\$ 1 ; 8 \%)$. This apperars to be an error.; of the total SCAR) were modest contributors to the total SCAR. Assuming an average antibiotic cost of $\$ 20$, the total SCAR attributable to each ambulatory antibiotic prescription would increase antibiotic costs by $65 \%$ (range: 15-475 \%) if incorporated into antibiotic costs paid by patients or payers.
\end{abstract}

Conclusions: Each ambulatory antibiotic prescription is associated with a hidden SCAR that substantially increases the cost of an antibiotic prescription in the United States. This finding raises concerns regarding the magnitude of misalignment between individual and societal antibiotic costs.

Keywords: Societal costs, Antibiotic resistance, Primary care, Stewardship, Negative externality

\section{Background}

Antibiotic resistance is a major public health problem, with more than 2,000,000 infections and 23,000 deaths caused by antibiotic-resistant organisms annually in the United States [1]. Most antibiotic use occurs in the ambulatory setting where it contributes to the development of antibiotic resistance [2, 3], which in turn increases health care costs $[1,4-7]$.

\footnotetext{
* Correspondence: cmichaelidis@partners.org

${ }^{1}$ Division of General Medicine and Primary Care, Brigham and Women's

Hospital, Harvard Medical School, Boston, MA, USA

${ }^{5}$ PGY-3, Internal Medicine, Brigham and Women's Hospital, 75 Frances Street, Boston, MA 02115, USA

Full list of author information is available at the end of the article
}

The relationship between ambulatory antibiotic prescribing, antibiotic resistance and increased health care costs is complex and likely influenced by heterogeneity in antibiotic use, bacterial response to antibiotic pressure, geographic distribution of resistance genes, non-human reservoirs of antibiotic resistance and impact of bacterial resistance on clinical outcomes. At a fundamental level, however, the association between ambulatory antibiotic prescribing, antibiotic resistance and increased health care costs implies a downstream societal cost of antibiotic resistance (SCAR) attributable to each ambulatory antibiotic prescription. The SCAR represents a hidden cost that, to our knowledge, has not been estimated at the prescription 
level and could (1) help quantify the economic burden of antibiotic resistance linked to ambulatory antibiotic prescribing, (2) inform future cost and cost-effectiveness analyses pertaining to ambulatory antibiotic prescribing, and (3) frame policy discussions regarding stewardship efforts.

Antibiotic resistance increases societal costs by increasing health care utilization, shifting antibiotic use towards more costly second-line agents, and leading to the development of antibiotic stewardship programs $[1,2]$. In this exploratory analysis, we estimated the total SCAR attributable to each ambulatory antibiotic prescription in the U.S. by assuming that the total SCAR is the sum of the incremental societal costs of hospitalization, secondline inpatient antibiotic use, second-line outpatient antibiotic use, and antibiotic stewardship associated with antibiotic resistance. The goal of this exploratory analysis was to provide an initial estimate of the total annual incremental SCAR in the U.S. attributable to each ambulatory antibiotic prescription that was simple, valid and conservative, while accounting for uncertainty in sensitivity analyses. The goal of this analysis was not to quantify all potential downstream costs and benefits of ambulatory antibiotic prescribing but rather to investigate and estimate poorly understood downstream SCAR attributable to ambulatory antibiotic prescribing,

\section{Methods}

We developed four methods to estimate the components of the SCAR attributable to each antibiotic prescription, with each method focusing on a different mechanism by which antibiotic resistance increases societal costs. These four methods estimated the incremental costs of antibiotic resistance associated with (1) hospitalization, (2) secondline inpatient antibiotic use, (3) second-line outpatient antibiotic use, and (4) antibiotic stewardship. For all methods, we estimated the total annual incremental SCAR relative to a hypothetical "no antibiotic resistance" base case scenario.

Given the complex and poorly understood relationship between ambulatory antibiotic prescribing and increased health care costs and the exploratory nature of this modeling analysis, we assumed that each human ambulatory antibiotic prescription contributed equally to the total incremental costs of antibiotic resistance associated with the four methods mentioned above and widely varied all model parameters in sensitivity analyses. If broad-spectrum antibiotics contribute more than narrow-spectrum antibiotics to the downstream social costs of ambulatory antibiotic prescriptions, this assumption would potentially over-estimate the downstream SCAR for narrow-spectrum antibiotics and under-estimate the downstream SCAR for broad-spectrum agents. To estimate this impact, we performed a secondary sensitivity analysis in which we arbitrarily assumed that broad-spectrum antibiotics were associated with double the impact of narrow-spectrum antibiotics on the total incremental SCAR attributable to each antibiotic prescription. For each method, we estimated the annual SCAR in the U.S. attributable to the specific cost mechanism of interest and then divided this estimate by the annual number of ambulatory antibiotic prescriptions in the U.S. (263 million, range: 213-263 million) [8], after adjusting for the percentage, by weight, of antibiotics prescribed to animals ( $80 \%$, range: $75-85 \%$ ) [9], the relative weighting of antibiotic use in humans versus animals on human antibiotic resistance costs (1:1, range: $1: 1-3: 1)$, and the percentage of antibiotics prescribed to humans in the inpatient setting (20\%; range: $10-30 \%$ ) [3]. We adjusted for antibiotic use in animals in light of the evidence linking such use to infections with antibioticresistant pathogens in humans [10]. In the absence of data, we assumed that human and animal antibiotic use had equal impact on human antibiotic resistance costs, an assumption that biases the analysis towards lower cost estimates. Within each method, we varied all parameters across wide ranges in sensitivity analyses. All costs are reported in 2013 US\$ with prior years' costs inflated using the U.S. Consumer Price Index.

We then summed the individual cost components to obtain an estimate for the total SCAR attributable to each ambulatory antibiotic prescription. This approach is conservative because it assumes that all societal costs of antibiotic resistance are captured in these four estimates.

\section{SCAR Component \#1: Hospitalization Costs}

Using a previously described approach [11], we estimated the SCAR attributable to each antibiotic prescription arising from the incremental societal costs of hospitalizations for antibiotic-resistant infections relative to antibioticsusceptible infections (Table 1). Briefly, this approach allocates a prior estimate of the total U.S. annual incremental hospitalization cost due to antibiotic resistant infections ( $\$ 41$ billion, range $\$ 6-60$ billion) [12-14] across the total annual number of ambulatory antibiotic prescriptions in the U.S. (263 million, range: 213-263 million) [8] after adjusting for animal and inpatient antibiotic use as described above. Although initially used to estimate the SCAR attributable to antibiotic use for acute respiratory tract infections, this approach can be generalized to all infections because it assumes equal impact of each ambulatory antibiotic prescription on societal costs of hospitalization for antibiotic-resistant infections. In the base case, we assumed that the annual incremental SCAR due to hospitalizations for antibiotic-resistant infections in the U.S. equaled \$41 billion in 2013 US\$ based on an estimate of annual hospitalization costs attributable to antibioticresistant infections relative to no infections in adults at a Chicago teaching hospital [12] extrapolated to a national 
Table 1 Parameters used to estimate the hidden societal cost of antibiotic resistance (SCAR) attributable to each ambulatory antibiotic prescription in the U.S. arising from four cost components

\begin{tabular}{|c|c|c|c|}
\hline Parameters & Base Case & Range & Source \\
\hline \multicolumn{4}{|l|}{ SCAR Component \#1: Hospitalization Cost } \\
\hline Annual hospitalization costs due to resistant infections (US\$, millions) & 41,000 & $6,000-60,000$ & $12-14$ \\
\hline \multicolumn{4}{|l|}{ Percentage of annual hospitalization costs due to incremental costs } \\
\hline of antibiotic-resistant versus -susceptible infections (\%) & 35 & $25-45$ & 15,16 \\
\hline \multicolumn{4}{|l|}{ SCAR Component \#2: Second-Line Inpatient Antibiotic Cost } \\
\hline \multicolumn{4}{|l|}{ Annual antibiotic costs for resistant infections (US\$, millions) } \\
\hline Linezolid & 743 & $594-892$ & 17 \\
\hline Daptomycin & 713 & $570-856$ & 18 \\
\hline Carbapenems & 474 & $379-569$ & 19 \\
\hline Vancomycin & 292 & $234-350$ & 19 \\
\hline Tigecycline & 148 & $118-178$ & 20 \\
\hline Ceftaroline & 88 & $58-118$ & 21 \\
\hline \multicolumn{4}{|l|}{ Annual U.S. antibiotic costs for susceptible infections as } \\
\hline a percentage of costs for resistant infections (\%) & 30 & $10-50$ & 22 \\
\hline
\end{tabular}

SCAR Component \#3: Second-Line Outpatient Antibiotic Cost

Antibiotic mix in the resistance scenario (\%)

Narrow-spectrum penicillins

$16-24$

23,24

First-generation cephalosporins

$7-11$

23,24

Sulfonamides

$5-11$

23,24

Tetracyclines

$5-7$

23,24

Macrolides

$17-23$

23,24

Quinolones

$15-19$

23,24

Broad-spectrum cephalosporins

8-10

23,24

Broad-spectrum penicillins

$8-10$

23,24

Lincomycin derivatives

$1-3$

23,24

Antibiotic mix in the no resistance scenario (\%)

Narrow-spectrum penicillins

First-generation cephalosporins

23,24 Estimate

Sulfonamides

$12-16$

23,24 Estimate

Tetracyclines

$11-15$

23,24 Estimate

Macrolides

$7-11$

23,24 Estimate

Average cost per antibiotic class (US\$)

Narrow-spectrum penicillins (penicillin)

First-generation cephalosporins (cephalexin)

Sulfonamides (trimethoprim-sulfamethoxazole)

Tetracyclines (doxycycline)

Macrolides (azithro, clarithro and erythromycin)

Quinolones (cipro, levo and moxifloxacin)

Broad-spectrum cephalosporins (cefuroxime, cefdinir)

26, Estimate

26, Estimate 
Table 1 Parameters used to estimate the hidden societal cost of antibiotic resistance (SCAR) attributable to each ambulatory antibiotic prescription in the U.S. arising from four cost components (Continued)

\begin{tabular}{|c|c|c|c|}
\hline \multicolumn{4}{|l|}{ SCAR Component \#4: Antibiotic Stewardship Cost } \\
\hline Acute care hospital beds, U.S. (thousands) & 924 & Not varied & 28 \\
\hline \multicolumn{4}{|l|}{ Staff full time equivalents, stewardship program per 200 beds } \\
\hline Pharmacist & 1.0 & $0.3-2.0$ & 29 \\
\hline Physician & 0.25 & $0.0-1.0$ & 29 \\
\hline \multicolumn{4}{|l|}{ Annual salary (\$US) } \\
\hline Pharmacist, Infectious Diseases & 120,000 & $100,000-140,000$ & 30 \\
\hline Physician, Infectious Diseases & 190,000 & $150,000-230,000$ & 31 \\
\hline Salary multiplier for fringe benefits (\%) & 120 & $110-130$ & Estimate \\
\hline Annual educational costs per 200 beds (\$US) & 15,000 & $10,000-20,000$ & Estimate \\
\hline \multicolumn{4}{|l|}{ SCAR Components \#1-4 } \\
\hline Antibiotics prescribed to humans, by weight (\%) & 20 & $15-25$ & 9 \\
\hline $\begin{array}{l}\text { Relative weighting of human versus animal antibiotic use } \\
\text { on human antibiotic resistance costs }\end{array}$ & $1: 1$ & $1: 1-3: 1$ & Estimate \\
\hline Antibiotics prescribed in ambulatory setting (\%) & 80 & $70-90$ & 3 \\
\hline Annual ambulatory antibiotic prescriptions (millions) & 263 & $213-313$ & 8 \\
\hline
\end{tabular}

level [13]. This prior study used multiple linear regression methods to estimate the incremental costs of antibioticresistant infections relative to no infections in a hospitalized adult cohort after controlling for age, illness severity and exposure to intensive care unit, surgery and hospitalacquired infection [12]. The study included both community and hospital-acquired antibiotic resistant infections. The range (\$6-60 billion) around this point estimate represents a wide and conservative estimate based on reported result variation [12].

Because this prior study estimated the total incremental societal cost of hospitalization for antibiotic-resistant infections relative to no infections rather than relative to antibiotic-susceptible infections, it potentially overestimates the incremental costs of antibiotic-resistant infections relative to antibiotic-susceptible infections. To correct for this potential overestimate, we assumed that only $35 \%$ (range: 25-45 \%) of the total incremental societal costs of hospitalization for antibiotic-resistant infections could be attributable to antibiotic resistance based on estimates of the incremental costs of antibiotic-resistant versus antibiotic-susceptible Staphylococcal and Enterococcal hospital infections $[15,16]$.

SCAR Component \#2: Second-Line Inpatient Antibiotic Costs To estimate the SCAR attributable to each antibiotic prescription arising from the incremental cost of secondline inpatient antibiotic use, we assumed that this cost equaled the total annual incremental U.S. cost of antibiotics used, in the inpatient setting, primarily for the treatment of antibiotic-resistant infections (carbapenems, ceftaroline, daptomycin, linezolid, tigecycline and vancomycin) relative to antibiotics used primarily for the treatment of antibiotic-susceptible infections (Table 1) [17-21]. The above antibiotics were selected by authors in an effort to select those agents most likely to be used in the setting of concern for antibiotic resistance and informed by a discussion of recent antibiotic development [1]. We conservatively did not include the aminoglycosides given some concern that utilization may not predominantly reflect concern for resistance. Given that inpatient aminoglycoside use accounts for only $1.8 \%$ of total inpatient antibiotic use and $\$ 71$ million in annual costs, this modeling choice would be unlikely to materially impact results [17]. Annual U.S. antibiotic cost data were drawn from the IMS Health National Sales Perspective database $[17,19]$ and manufacturer quarterly financial reports [18, $20,21]$. We assumed that $100 \%$ of the annual cost of each of the aforementioned antibiotics and $0 \%$ of the annual cost of all other antibiotics were associated with the treatment of antibiotic-resistant infections in the inpatient setting. We assumed that antibiotic-susceptible infections would have occurred in the absence of antibiotic resistant-infections and that antibiotic costs for treatment of antibiotic-susceptible infections were $30 \%$ (range: $10-50 \%$ ) of antibiotic costs for treatment of resistant infections based on decreased overall antibiotic costs for patients hospitalized with methicillin-susceptible versus methicillin-resistant Staphylococcus aureus infections [22]. We did not consider antibiotic development costs in our estimates due to concerns that doing so could result in double-counting of societal costs. 


\section{SCAR Component \#3: Second-Line Outpatient Antibiotic Costs}

To estimate the SCAR attributable to each antibiotic prescription arising from the incremental societal costs of second-line outpatient antibiotic use, we assumed this cost was a function of incremental antibiotic costs in the presence versus absence of antibiotic resistance (Table 1). In both the "resistance" and "no resistance" scenarios, we assumed 263 million (range: $213-313$ million) ambulatory antibiotic prescriptions annually in the U.S. based on IMS Health pharmacy-level data for dispensed antibiotic prescriptions [8]. To define the antibiotic mix in the "resistance" scenario, we assumed that the current mix of narrow- and broad-spectrum antibiotics reported in the NAMCS/NHAMCS data for pediatric [23] and adult [24] populations reflects physician prescribing behavior in the setting of concern for antibiotic resistance. To define the antibiotic mix in the "no resistance" scenario, we assumed that use of broad-spectrum antibiotics (broad-spectrum penicillins and cephalosporins, quinolones and lincomycin derivatives) reflects concern for antibiotic-resistant pathogens and would not have occurred in the absence of antibiotic resistance. In this scenario, we assumed that $100 \%$ of broad-spectrum antibiotics would be replaced by narrow-spectrum antibiotics (narrow-spectrum penicillins, first-generation cephalosporins, macrolides, sulfonamides, tetracyclines) in the same volume-weighted ratios reported in the NAMCS/NHAMCS data [23, 24]. Using a previously described approach [25], we estimated antibiotic costs using prices from an online pharmacy database [26] and not average wholesale prices (AWPs) due to concerns that AWPs do not accurately reflect acquisition costs [27]. Because this online pharmacy database only includes costs for antibiotics commonly prescribed in the ambulatory setting, we were unable to use this database for the second-line inpatient antibiotic cost estimation method described above. For each antibiotic class, we assumed that the class included only commonly used agents within that class (Table 1).

We assumed that antibiotic resistance caused only an increase in cost per ambulatory antibiotic prescription and not an increase in the number of ambulatory antibiotic prescriptions per patient for a given infection or total duration of antibiotic therapy. This assumption is likely conservative given that ambulatory patients with antibiotic-resistant pathogens may return to clinic more frequently than those with antibiotic-susceptible pathogens and require multiple antibiotic prescriptions [7].

\section{SCAR Component \#4: Antibiotic Stewardship Costs}

To estimate the SCAR attributable to each antibiotic prescription arising from the incremental societal costs of antibiotic stewardship, we assumed that such costs would not have been incurred in the absence of concern for the potential development of antibiotic resistance and equaled the annual costs of antibiotic stewardship programs incurred by U.S. hospitals (Table 1). Given an absence of published literature providing detailed cost data for such programs, we extrapolated the annual costs of antibiotic stewardship programs from the annual costs of antibiotic stewardship programs per acute care bed $[28,29]$. Based on guidelines from the Infectious Disease Society of America, we assumed that the salary costs for infectious disease trained physicians ( 0.25 full time equivalent per 200 beds, range: $0.0-1.0)$ and pharmacists (1.0 full time equivalent per 200 beds, range: 0.3-2.0) represented the primary costs of such programs [29-32]. In addition, because educational activities are a core element of antibiotic stewardship programs, we estimated annual costs of educational activities of $\$ 15,000$ (range: \$10,000-20,000) per 200 beds, assuming that 30 physicians would attend 24-h educational activities annually at an average salary cost of $\$ 120$ per hour [31]. This estimate is conservative in that it does not include costs of collaboration with clinical microbiologists, infection control officers, hospital epidemiologists, or information technology specialists [32]. Furthermore, this estimate does not include physician time costs incurred when interfacing with antibiotic stewardship programs that include formulary restriction and pre-authorization as core program components [32].

\section{Results}

When we summed the costs of all four components, the SCAR attributable to each ambulatory antibiotic prescription was \$13 in the base case and ranged from \$3-\$95 in sensitivity analyses (Table 2). The greatest contributor to the base case total SCAR estimate was the cost of hospitalization (69 \%; base case: \$9, range: $\$ 1-\$ 39)$. The costs of second-line inpatient antibiotic use (8\%; base case: $\$ 1$, range: $\$ 0-\$ 4)$, second-line outpatient antibiotic use (15\%; base case: $\$ 2$, range: $\$ 2-\$ 47)$ and

Table 2 Estimate of the hidden societal cost of antibiotic resistance (SCAR) attributable to each ambulatory antibiotic prescription in the U.S. arising from four cost components

\begin{tabular}{lll}
\hline SCAR Component & \multicolumn{2}{l}{ Cost Estimates } \\
\cline { 2 - 3 } & Base Case & Range \\
\hline \#1: Hospitalization Cost & $\$ 9$ & $\$ 1-\$ 39$ \\
\#2: Second-line Inpatient Antibiotic Cost & $\$ 1$ & $\$ 0-\$ 4$ \\
\#3: Second-line Outpatient Antibiotic Cost & $\$ 2$ & $\$ 2-\$ 47$ \\
\#4: Antibiotic Stewardship Cost & $\$ 1$ & $\$ 0-\$ 5$ \\
Total SCAR Attributable to Each Antibiotic $^{\text {Prescription }}{ }^{\text {a }}$ & $\$ 13$ & $\$ 3-\$ 95$ \\
\hline
\end{tabular}

${ }^{\mathrm{a}}$ Sum of the four cost components above, rounded to nearest SUS 
antibiotic stewardship ( $8 \%$; base case: $\$ 1$, range: $\$ 0-\$ 5$ ) were modest contributors to the total SCAR attributable to each antibiotic prescription. Assuming an average antibiotic cost of $\$ 20$, the total SCAR attributable to each ambulatory antibiotic prescription would increase antibiotic costs by $65 \%$ (range: 15-475\%) if incorporated into antibiotic costs experienced by patients or payers.

In the secondary sensitivity analysis in which we assumed that a broad-spectrum antibiotic prescription was associated with double the impact of narrow-spectrum antibiotic prescription on the total incremental SCAR attributable to each ambulatory antibiotic prescription, we estimated that the total downstream SCAR attributable to each ambulatory antibiotic prescription was $\$ 9$ for a narrow-spectrum antibiotic prescription and $\$ 17$ for a broad-spectrum antibiotic prescription.

Assuming that there are 263 million ambulatory antibiotic prescriptions annually in the United States [8] and that $30 \%$ of all ambulatory antibiotic prescribing is inappropriate [33], our base case estimate for the total SCAR attributable to each ambulatory antibiotic prescription (\$13) suggests that total and inappropriate ambulatory antibiotic prescribing account for \$3.4 billion and $\$ 1.0$ billion, respectively, in SCAR annually in the United States.

\section{Discussion}

This exploratory analysis quantifies the hidden, but considerable, societal cost of ambulatory antibiotic prescribing. We conservatively estimated that the hidden SCAR attributable to each ambulatory antibiotic prescription is $\$ 13$ in the base case and substantially increases the societal cost of an antibiotic prescription if incorporated into antibiotic costs paid by patients or payers.

This estimate has important implications for patients, clinicians, payers and policy makers. For patients and clinicians, this study further raises the costs of inappropriate antibiotic prescribing for diagnoses such as the common cold, upper respiratory tract infections, and acute bronchitis, for which antibiotics are commonly prescribed $[34,35]$ despite evidence that the patient benefit is virtually zero [36, 37] and of harm is moderate [38]. For conditions such as community-acquired pneumonia, skin and softtissue infections and urinary tract infections where antibiotics more clearly provide benefit, this study has more limited implications. For policymakers, this study raises serious concerns regarding the risks of not considering the hidden SCAR attributable to ambulatory antibiotic prescribing and the optimal mechanism to appropriately allocate this economic burden. The major risk of ignoring the SCAR attributable to each ambulatory prescription is to perpetuate a misalignment because individual and societal costs that encourages excess antibiotic use to that which would be expected if societal costs were considered in a perfect market [39, 40].

Classically, economists have proposed several solutions to address such a 'negative externality'-regulation, taxation or permits, all of which have been considered as solutions to ease the economic burden of antibiotic resistance attributable to antibiotic use [40]. These cost allocation mechanisms, however, assume that there is a single antibiotic consumer in the U.S. healthcare system who should bear this economic burden. Future discussion of the appropriate bearer of this economic burden and the optimal cost allocation mechanism must be informed by an understanding of the magnitude of the economic burden and the likely impact of any cost allocation mechanism on health outcomes. This study did not attempt to quantify all potential downstream costs and benefits of ambulatory antibiotic prescribing but rather one poorly understood downstream cost of ambulatory antibiotic prescribing, namely the costs of antibiotic resistance. In estimating the economic burden of antibiotic resistance associated with ambulatory antibiotic prescribing, this analysis provides one element necessary for an important discussion regarding net costs and benefits of ambulatory antibiotic prescribing.

This study has several strengths. First, this study employed a novel approach to estimate the hidden SCAR attributable to each ambulatory antibiotic prescription. Second, this study incorporated conservative assumptions that biased the analysis in favor of lower cost estimates. Within the four estimation methods, we focused only on major cost drivers. Further, when summing across the four estimation methods, we assumed that they represented all of the mechanisms by which antibiotic resistance increases societal costs. If there are other material mechanisms by which antibiotic use leads to SCAR, i.e. by leading to increased utilization of outpatient services, then this analysis would result in a conservative estimate of the total SCAR attributable to each ambulatory antibiotic prescription.

Our analysis also has limitations. First, in the absence of published data describing the relative impact of human and agricultural antibiotic use on antibiotic resistance in humans, all four estimation methods relied on our estimate of the relative impact of human versus animal antibiotic use on antibiotic resistance costs in humans. Our assumption that each unit weight of antibiotic use in humans and animals equally impacts antibiotic-resistance costs in humans, however, is likely conservative. Second, in the absence of available data allowing us to make reasonable estimates regarding the relative contribution of different antibiotics (i.e. amoxicillin relative to amoxicillinclavulanate or first-generation cephalosporins relative to quinolones), we assumed that each human ambulatory antibiotic prescription contributed equally to the total 
incremental SCAR, which may potentially over-estimate the downstream SCAR for narrow-spectrum antibiotics and under-estimate the downstream SCAR for broadspectrum antibiotics are greater contributors to the total incremental SCAR than narrow-spectrum antibiotics. Third, in the absence of published data providing further guidance, our four estimation methods did not account for antimicrobial prescribing in long-term inpatient care facilities, potential contribution of inappropriate antibiotic dosing and duration and antibiotic resistance introduced from travel exposures outside of the United States. Fourth, each method had unique limitations. In the second-line inpatient antibiotic cost method, we estimated the SCAR attributable to ambulatory antibiotic prescribing based in part on antibiotics that are predominantly used for treatment of antibiotic resistant infections in the hospital setting (i.e. carbapenems, daptomycin, etc.). Further, some proportion of second-line antibiotic use may be inappropriate and not reflective of concern for antibiotic resistant infections, which could lead to an overestimate of the SCAR arising from this source. In the second-line outpatient antibiotic cost method, we assumed that ambulatory use of broad-spectrum antibiotics predominantly reflects physician concern for antibiotic resistance when it may also reflect other attitudes towards the value of specific antibiotics. In the antibiotic stewardship cost method, we estimated total U.S. costs of antibiotic stewardship by extrapolating costs of stewardship from a 200-bed community hospital to a national level based on bed size, assuming a linear relationship between bed-size and stewardship costs across all hospitals [28, 29]. Finally, these estimates are unlikely to be generalizable outside of the U.S. given different antibiotic utilization, resistance and costs.

\section{Conclusions}

In summary, we found that there is a considerable hidden SCAR attributable to each ambulatory antibiotic prescription that would substantially increase the total societal costs of ambulatory antibiotic prescribing if incorporated into costs experienced by payers or patients. The magnitude of the misalignment between antibiotic costs experienced on individual and societal levels reinforces the need to develop and validate innovative strategies to appropriately align individual and societal costs of antibiotic use in an effort to safely reduce total societal costs of antibiotic use.

\section{Abbreviation \\ SCAR: Societal cost of antibiotic resistance}

\section{Acknowledgments}

Not applicable.

Funding

This work was supported by the National Institutes of Health [grant numbers T32AG21885 to CIM, RC4 AG039115 to JAL, KL2TR000146 to RKS], the
National Institute of Allergy and Infectious Diseases [grant numbers R21 Al097759 to JAL, R01Al076256 to KJS], the Agency for Healthcare Research and Quality [grant number R18 HS018419 to JAL], the Doris Duke Charitable Foundation, and the University of Pittsburgh School of Medicine Clinical Scientist Training Program.

\section{Availability of data and materials}

All data within this manuscript were obtained from publically available sources as cited above with all central assumptions described in Table 1.

\section{Authors' contributions}

All authors listed have made substantial contributions to this work. CIM and KJS performed the primary analysis. CIM drafted the initial manuscript. CIM, MJF, CJL, JAL, MPN, RKS, RKZ and KJS all made substantial contributions to the conception, design and final manuscript. All authors have reviewed and approved the manuscript.

\section{Competing interests}

$\mathrm{CJ}$ and MPN reported receiving research grants from Medlmmune, Merck, Pfizer and Sanofi Pasteur and consulting for Medlmmune. RKS reported receiving research grants from Astellas and Merck. RKZ reported receiving research grants from Merck, Pfizer and Sanofi Pasteur. All other authors report no conflicts of interest.

\section{Consent for publication}

Not applicable.

\section{Ethics approval and consent to participate}

This manuscript is based on a secondary analysis of publically available data. Thus, no novel participant-level was obtained and thus no informed consent was required. This manuscript was considered exempt from requiring approval from the Institutional Review Board of Brigham and Women's Hospital.

\section{Author details}

'Division of General Medicine and Primary Care, Brigham and Women's Hospital, Harvard Medical School, Boston, MA, USA. ${ }^{2}$ Division of General Internal Medicine, University of Pittsburgh School of Medicine, Pittsburgh, PA USA. ${ }^{3}$ Center for Health Equity Research and Promotion, VA Pittsburgh Healthcare System, Pittsburgh, PA, USA. ${ }^{4}$ Department of Family Medicine, University of Pittsburgh School of Medicine, Pittsburgh, PA, USA. ${ }^{5}$ PGY-3, Internal Medicine, Brigham and Women's Hospital, 75 Frances Street, Boston, MA 02115, USA.

Received: 24 March 2016 Accepted: 28 October 2016

Published online: 08 November 2016

\section{References}

1. Centers for Disease Prevention and Control. Antibiotic resistance threats in the United States. http://www.cdc.gov/drugresistance/threat-report-2013. Accessed 12 Sept 2016.

2. Institute of Medicine. The resistance phenomenon in microbes and infectious disease vectors: implications for human health and strategies for containment. Workshop summary. Washington DC: The National Academies Press; 2003.

3. Wise $\mathrm{R}$, Hart $\mathrm{T}$, Cars $\mathrm{O}$, et al. Antimicrobial resistance is a major threat to public health. BMJ. 1998;317:609-10.

4. Goosens H, Ferech M, Stichele RV, et al. Outpatient antibiotic use in Europe and association with resistance: a cross-national database study. Lancet. 2005;365:579-87.

5. van de Sande-Bruinsma N, Grundman H, Verloo D, et al. Antimicrobial drug use and resistance in Europe. Emerg Infect Dis. 2008;14:1722-30.

6. Donnan PT, Wei L, Steinke DT, et al. Presence of bacteriuria caused by trimethoprim resistant bacteria in patients prescribe antibiotics: multilevel model with practice and individual patient data. BMJ. 2004;328:1297-301.

7. Butler C, Hillier S, Roberts Z, et al. Antibiotic-resistant infections in primary care are symptomatic for longer and increase workload: outcomes for patients with E. coli UTIs. Brit J Gen Pract. 2006;56:686-92.

8. Hicks LA, Bartoces MG, Roberts RM, et al. US outpatient antibiotic prescribing variation according to geography, patient population, and provider specialty in 2011. Clin Infect Dis. 2015;60:1308-16. 
9. Pew Charitable Trust. Record-high antibiotic sales for meat and poultry production. http://www.pewtrusts.org/en/multimedia/data-visualizations/ 2013/recordhigh-antibiotic-sales-for-meat-and-poultry-production. Accessed 12 Sept 2016

10. Marshall BM, Levy SB. Food animals and antimicrobials: impacts on human health. Clin Microbiol Rev. 2011;24:718-33.

11. Michaelidis Cl, Zimmerman RK, Nowalk MP, Fine MJ, Smith KJ. Cost-effectiveness of procalcitonin-guided therapy for outpatient management of acute respiratory tract infections in adults. J Gen Intern Med. 2014;29:579-86.

12. Roberts RR, Hota B, Ahmad I, et al. Hospital and societal costs of antimicrobial-resistant infections in a Chicago teaching hospital: implications for antibiotic stewardship. Clin Infect Dis. 2009;49:1175-84.

13. Bush K, Courvalin P, Dantas G, et al. Tackling antibiotic resistance. Nat Rev Microbiol. 2011;9:894-6.

14. Institute of Medicine. Antimicrobial resistance: issues and options. Workshop report. Washington DC: The National Academy Press; 1998.

15. Cosgrove SE. The relationship between antimicrobial resistance and patient outcomes: mortality, length of hospital stay, and health care costs. Clin Infect Dis. 2006:42:582-89.

16. Carmeli Y, Eliopoulos G, Mozaffari G, et al. Health and economic outcomes of vancomycin-resistant Enterococci. Arch Intern Med. 2002;162:2223-8.

17. Suda KJ, Hicks LA, Roberts RM, et al. A national evaluation of antibiotic expenditures by healthcare setting in the United States, 2009. J Antimicrob Chemother. 2013;68:715-8.

18. Business Wire. Cubist Reports Fourth Quarter and Full Year 2012 Financial Results. http://www.businesswire.com/news/home/20130123006397/en/ Cubist-Reports-Fourth-Quarter-Full-Year-2012. Accessed 12 Sept 2016.

19. Wenzel R, Bate $G$, Kirkpatrick P. Fresh from the pipeline: tigecycline. Nat Rev Drug Discov. 2005;4:809-10.

20. Pfizer. Fourth Quarter Financial Report 2012. Available from: http://www pfizer.com/files/investors/presentations/q4performance_012913.pdf. Accessed 12 Sept 2016.

21. Forest-Laboratories Third Quarter 2013 Sales. Available from http://www businesswire.com/news/home/20130115005512/en/Forest-LaboratoriesReports-Fiscal-Year-2013-Quarter. Accessed 12 Sept 2016.

22. Inan D, Saba R, Gunseron F, et al. Daily antibiotic cost of nosocomial infections in a Turkish university hospital. BMC Infect Dis. 2005;5:5.

23. Hersh AL, Shapiro DJ, Pavia AT, et al. Antibiotic prescribing in ambulatory pediatrics in the United States. Pediatrics. 2011;128:1053-61.

24. Shapiro DJ, Hicks LA, Pavia AT, et al. Antibiotic prescribing for adults in ambulatory care in the USA, 2007-09. J Antimicrob Chemother. 2014;69:234-40.

25. Kouta I, Elkin E, Blinder $\mathrm{V}$, et al. Cost-effectiveness of full coverage of aromatase inhibitors for Medicare beneficiaries with early breast cancer. Cancer. 2013;119:2494-502.

26. GoodRx. Search Drug Prices. Available from: http://www.goodrx.com. Accessed 12 Sept 2016.

27. Curtis FR, Lettrich P, Fairman KA. What is the price benchmark to replace average wholesale price (AWP)? J Manag Care Pharm. 2010;16:492-501.

28. American Hospital Association. Fast Facts on American Hospitals. http://www.aha.org/research/rc/stat-studies/101207fastfacts.pdf. Accessed 12 Sept 2016.

29. Carling P, Fung T, Killion A, et al. Favorable impact of a multidisciplinary antibiotic management program conducted during 7 years. Infect Control Hosp Epidemiol. 2003;24:699-706.

30. Salary.com. Clinical Pharmacist Annual Salary. http://swz.salary.com/ SalaryWizard/Clinical-Pharmacist-Salary-Details.aspx. Accessed 12 Sept 2016.

31. Salary.com. Infectious Disease Physician Annual Salary. http://swz.salary. com/SalaryWizard/Physician-Infectious-Disease-Salary-Details.aspx. Accessed 12 Sept 2016

32. Dellit TH, Owens RC, McGowan JE, et al. Infectious Diseases Society of American and the Society for Healthcare Epidemiology of American guidelines for developing an institutional program to enhance antimicrobial stewardship. Clin Infect Dis. 2007:44:159-77.

33. Fleming-Dutra KE, Hersh AL, Shapiro DJ, et al. Prevalence of inappropriate antibiotic prescriptions among US ambulatory care visits, 2010-2011. JAMA. 2016:315(17):1864-73.

34. Gonzales R, Malone DC, Maselli JH, et al. Excessive antibiotic use for acute respiratory infections in the United States. Clin Infect Dis. 2001;33:757-62.

35. Gonzales R, Steiner JF, Sande MA. Antibiotic prescribing for adults with colds, upper respiratory tract infections, and bronchitis by ambulatory care physicians. JAMA. 1997;278:901-4
36. Kenealy T, Arrol B. Antibiotics for the common cold and acute purulent rhinitis. Cochrane Database SystRev. 2013:(6):CD000247.

37. Smith SM, Fahey T, Smucny J, et al. Antibiotics for acute bronchitis. Cochrane Database Syst Rev. 2014;(3):CD000245.

38. Linder JA. Antibiotic prescribing for acute respiratory infections-success that's way off the mark. JAMA. 2013;173:273-5.

39. Phelps T. Bug/drug resistance: sometimes less is more. Med Care. 1989;27:194-203.

40. Smith RD, Yago M, Millar M, et al. A macroeconomic approach to evaluating policies to contain antimicrobial resistance: a case study of methicillinresistant Staphylococcus aureus (MRSA). Appl Health Econ Health Policy. 2006;5:55-65.

\section{Submit your next manuscript to BioMed Central and we will help you at every step:}

- We accept pre-submission inquiries

- Our selector tool helps you to find the most relevant journal

- We provide round the clock customer support

- Convenient online submission

- Thorough peer review

- Inclusion in PubMed and all major indexing services

- Maximum visibility for your research

Submit your manuscript at www.biomedcentral.com/submit
) Biomed Central 